\title{
A New Protocol for Cooperative Spectrum Sharing in Mobile Cognitive Radio Networks
}

\author{
Seyed Alireza HOSSEINI ${ }^{1}$, Bahman ABOLHASSANI ${ }^{1}$, Seyed Mohammad Sajad SADOUGH ${ }^{2}$ \\ ${ }^{1}$ School of Electrical Engineering, Iran University of Science and Technology, 16197, Tehran, Iran \\ ${ }^{2}$ Cognitive Telecommunication Research Group, Dept. of Electrical Engineering, Faculty of Electrical Engineering, \\ Shahid Beheshti University G. C., 1983963113, Tehran, Iran
}

arhoseini@iust.ac.ir, abolhassani@iust.ac.ir, s_sadough@sbu.ac.ir

\begin{abstract}
To optimize the usage of limited spectrum resources, cognitive radio $(C R)$ can be used as a viable solution. The main contribution of this article is to propose a new protocol to increase throughput of mobile cooperative CR networks (CRNs). The key challenge in a CRN is how the nodes cooperate to access the channel in order to maximize the CRN's throughput. To minimize unnecessary blocking of CR transmission, we propose a so-called new frequency-range MAC protocol (NFRMAC). The proposed method is in fact a novel channel assignment mechanism that exploits the dependence between signal's attenuation model, signal's frequency, communication range, and interference level. Compared to the conventional methods, the proposed algorithm considers a more realistic model for the mobility pattern of $C R$ nodes and also adaptively selects the maximal transmission range of each node over which reliable transmission is possible. Simulation results indicate that using NFRMAC leads to an increase of the total CRN's throughput by $6 \%$ and reduces the blocking rate by $10 \%$ compared to those of conventional methods.
\end{abstract}

\section{Keywords}

Blocking rate, cognitive radio, cooperative spectrum sharing, secondary users, spectrum sharing

\section{Introduction}

In wireless networks, spectrum allocation is conventionally performed by allocating a predefined portion of the spectrum to a primary (also referred to as licensed users) network for a long time and at a specific location [1]. In the past decade, the spectrum scarcity has become a real challenge such that static or fixed spectrum allocation is no longer appropriate. Recent measurements by Federal Communications Company (FCC) and other regulatory bodies indicate that primary users (PU) underutilize the licensed spectrum (also referred to as spectrum holes). Hence, recently, opportunistic and dynamic utilization of the spectrum has been advocated as a solution to cope with rising demand in unlicensed wireless services. Cognitive radio (CR) has been widely studied as an approach for increasing spectrum efficiency by allowing dynamic spec- trum access of unoccupied bands through spectrum sensing [2], [3]. In [4], the main tasks of a CR system are defined as i) spectrum sensing [5], ii) spectrum management [3], iii) spectrum mobility [6], and spectrum sharing [7], [8]. In CR terminology, unlicensed users are referred to as secondary users (SU) or cognitive users or secondary nodes. In opportunistic CR (also referred to as interweave CR) [9], whenever a PU is absent in the radio environment, SUs are allowed to use the frequency band; so CR should perform spectrum sensing to sense and estimate the presence of the PU [3], [9].

In this paper, we consider a transmission scenario where multiple CR users share the spectrum licensed with a PU. Moreover, we consider that CR users are mobile. Mobility may cause significant variation in the co-channel interference [10], [11] and make it a limiting factor. So, it is of great importance to propose an efficient and agile way for cooperative spectrum sharing among different cognitive users in such a scenario. In contrast to conventional networks where contiguous bands are typically occupied, a CR network operates over a set of widely-separated noncontiguous frequency bands. Communicating over such bands exhibits different RF attenuations that increase with the distance between the two users and also with the carrier frequency used for communication. It is hence necessary to propose efficient and fast algorithms for spectrum sharing that use a more realistic model for mobility of nodes and assign smaller channel frequencies to communicating nodes, which are farther from each other.

\subsection{Related Works}

Recently, different protocols have been presented for multi-channel medium access control (MAC) [12-15]. The authors in [13] have expanded a MAC protocol for the CR network (CRN) with a common control channel. By taking into account power limitation, this protocol simultaneously optimally allocates channel rate and power to CRs. The so called DC-MAC protocol in [14] is a distributed cross-layer for the sensing and allocation of spectrum. In [15], the internal heat model has been used for optimum selection of the spectrum and the transmission power for the secondary users. It is notable that the aforementioned protocols did not consider the dependence between RF attenuation (path 
loss) and frequency so that smaller frequencies makes less RF attenuations, and hence it should be assigned to nodes, which are farther from each other. These works however, are limited only to theoretical tasks in designing MAC layer without presenting details of algorithm implementations.

Reference [16] developed a distance-dependent MAC protocol (referred to as DDMAC). DDMAC is trying to increase the network's accessibility by considering dependence between the path loss and communication range as mentioned in previous paragraph. This model performs better compared to the best multichannel method (BMC) [11], [12], [17] which selects the best channel as that one having highest rate of data transmission.

However, in the above references, there are different limitations as described below. a) Except DDMAC method, other references have not considered nodes' mobility and also they have not considered the channel capacity limitation and the impact of channel's frequency in link stability. b) DDMAC method considered the maximum communication range (denoted $R_{c}$ ) as a fixed parameter. Obviously, fixed $R_{c}$ leads to a limited communication range while $R_{c}$ depends on the frequency used for radio transmission. So, the number of possible links can be increased if only we can increase the radius $R_{c}$ by assigning smaller frequencies for signal transmissions. c) The two DDMAC [17] and BMC [9] methods considered continuous time walking and did not consider a stop-time (stoppage time) in their random walking model [18], [19]. d) Finally, DDMAC method has not consider the network's throughput based on the number of CR users.

\subsection{Motivations and Contributions}

The main motivation of this paper is to overcome different limitations mentioned in Sec. 1.1. More precisely, our contribution is to propose a more general and realistic model for cooperative spectrum sharing in a mobile CRN.

As a first contribution, we propose a modified method for deriving parameter $R_{c}$ by proposing an adaptive rule that sets $R_{c}$ with respect to the received SINR and the instantaneous interference level at the CR receiver. More precisely, in the proposed method, each time a request to send (RTS) information (packets) is transmitted to the receiver, the receiver estimates interference level in free bands and sends it back to the transmitter with a Clear To Send (CTS) request. It is Notable that the DDMAC has used a constant maximum value for interference level, however it is necessary to consider interference level measured at the receiver, which can vary depending on the distance between two nods. Accordingly, it is more probable that the instantaneous interference would be less than the maximum interference in most of the times and locations of the network. Therefore in practice, $R_{C}$ which is SINR dependent is larger than $R_{c}$ considered in conventional DDMAC. In other words, by increasing $R_{C}$, adaptively when channel is good the probability that a demanded link is established would increase. i.e., we have

$$
\text { if } R_{c 2}>R_{c 1} \Rightarrow P\left(\operatorname{link} \mid R_{c 2}\right)>P\left(\operatorname{link} \mid R_{c 1}\right) \text {. }
$$

As a second contribution, we propose our NFRMAC protocol that assumes the stop-time for mobile nodes in the model. More precisely, in the random walk model that the previous references [12], [17] have considered, no stop time has been considered for the nodes. In practical networks, nodes can usually have a brief stops and are not in motion all the time. We will see that the throughput of the whole network is increased by considering stop times for the nodes, because it reduces the probability of primary and secondary interferences or at least keeps this probability unchanged. Thus, the model we consider here is more realistic, i.e., we consider the random walk model with adjustable stop (dwell) time. Obviously, considering appropriate stop time for the nodes causes the simulation conditions to be closer to real conditions. The effect of number of secondary users on the total throughput of the network and the blockage rate of packets is also considered in this paper.

The rest of this paper is organized as follows. The network model and the proposed algorithm's requirements are presented in Sec. 2. The proposed method is presented in Sec. 3. Parameters characterizing our network environment and conditions are presented in Sec. 4. Comparative simulation results that compare the proposed method with state of the art methods along with appropriate discussions are provided in Sec. 4.1. Finally, Section 5 concludes the paper.

\section{Network Model}

As depicted in Fig. 1, we consider a CR network in which each $\mathrm{CR}$ node can move with a random speed $0<v<6 \mathrm{~m} / \mathrm{s}$, or stay fixed in its location during a given stop-time period. The transmit power of each node $P_{t}$ is assumed to be fixed. The considered CR network and $M$ primary networks are located in the same geographical area. Figure 1 shows the above-mentioned scenario under condition with $M=2$. Both primary networks use two nonoverlapping equal bands. An Ad-hoc CR network with 40 moving nodes is considered in our simulations. There is no central controlling unit in this network and all nodes can communicate with their neighbors using a common control channel (CCC). For packet transmission, we use hand shaking protocol (RTS-CTS-AKC) [21], [22]. A link request is randomly made in this model and the number of source and destination nodes, their distances from each other and the interference levels at the receiver are not known before the link request is made. After each RTS, the two recent parameters (distance between sender and receiver and interference level) are estimated by the receiver and are sent to the sender with a controlling response of CTS. Connection is not possible between these two nodes if the required distance in CTS would be more than $R_{c}$ and consequently a blockage event occurs. However, connection would be possible for this link if $R_{C}$ is more than the required distance. Each CR user must be able to send over all allowable frequencies of primary users in simultaneous 


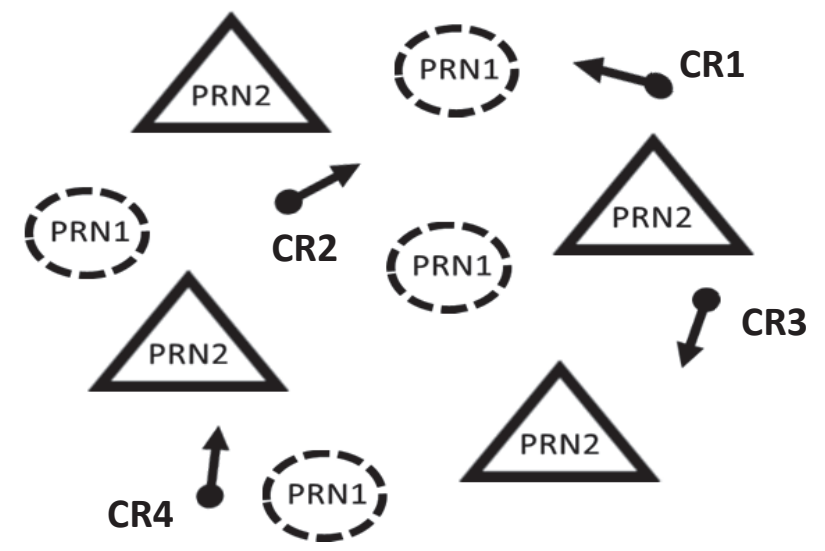

Fig. 1. The considered CRN with two primary networks in the same geographic and linking area. CR I, means: CR user I.

sending. To resolve this issue, each CR usually has a bank of fixed filters to gain this feature and by which it can select the desired sub-channel in each time period. Each CR has $1 \leq n_{t} \leq L$ transceivers, where $L$ is the number of frequencies of a primary network. Secondary users (SUs) cooperate with each other to maximize the throughput of the whole CRN. Cooperation of SUs refers to sharing local information (including SINR of receiving signal links and requested rate for the specific service) with other SUs.

In multi-channel MAC protocols, it is assumed that channel frequencies are determined as a constant parameter at the beginning of allocating a link and they remain unchanged during the linking procedure. However, it is probable that the operating frequency changes during the linking procedure of some of the protocols such as BMC and DDMAC. These two recent methods are more similar to our proposed method; thereby we compare our proposed method to these two methods in our simulations. In the BMC (best multi-channel) protocol, the first selected channel is the channel having the highest data rate.

\subsection{Path Loss Evaluation and the SINR}

In order to estimate the path loss and also for the channel model, we use a distance $d$ dependent path loss model between the transmitter and the receiver [23[, [24]. This model can be applied only for ranges of far fields i.e., distances of more than $d_{f}$ (reference distance or also called Fraunhofer distance). Parameter $d_{f}$ can be calculated using (1). In these equations, $D_{a}$ represents the maximum linear physical dimension of the antenna, $f$ refers to signal frequency, $C$ is the speed of light and $\lambda$ is the signal wavelength [24]. $P_{L}(f)$ also refers to path loss in frequency $f$ which depends on distance and frequency, and $n$ is the path loss order based on (2). The path loss exponent $n$ is considered to be 2 for free spaces and $3 \leq n \leq 4$ and even up to 6 , for big city spaces. These parameters are gathered and described in Tab. 1.

$$
d_{f}\left(f, D_{a}\right)=\max \left(D_{a}, \lambda, \frac{2 D_{a}^{2} f}{c}\right),
$$

$$
P L(d)=P L\left(d_{f}\right)\left(\frac{d}{d_{f}}\right)^{n} .
$$

The quality of a connection is usually determined by the signal-to-interference plus noise (SINR) ratio

$$
\text { SINR }=\frac{\text { Signal Power }}{\text { Noise }+ \text { Interference Power }} .
$$

\begin{tabular}{|c|c|}
\hline$N$ & Total number of channels of primary network \\
\hline$L$ & Number of channels for each PRN \\
\hline$n_{t}$ & Number of radios for each CR user \\
\hline$\mu^{*}$ & Communication range of nodes \\
\hline$R_{c}$ & No. of non-overlapping rings \\
\hline$m$ & CR transmit power \\
\hline$P_{t}$ & Path loss in channel $i$ \\
\hline$P_{L}\left(f_{i}\right)$ & Time duration of stopping by a node \\
\hline$t_{p a u s e}$ & Activity factor of primary network \\
\hline$\alpha_{i}$ & No. of primary networks \\
\hline$M$ & Interference (in dB) \\
\hline$I$ & Thermal noise power density \\
\hline$P_{t h}$ & density function \\
\hline$\lambda$ & Mean of packet rate generation for Poisson probability \\
\hline$D_{a}$ & Preferable channel list for node A \\
\hline$n$ & Distance between need A and B \\
\hline$\varphi_{i}(\mathrm{~A})$ & Node's speed \\
\hline$d_{A B}$ & \\
\hline$v$ & Path loss exponent \\
\hline
\end{tabular}

Tab. 1. The system parameters and their description.

Let $P_{t}$ be the transmitted power and $I$ the total power of the interfering signals and noise temperature in a subchannel, then $P_{L}(f, d)$ (path loss) is evaluated by (4).

$$
P_{L}(f, d)[\mathrm{dB}]=P_{t}[\mathrm{~dB}]-S I N R[\mathrm{~dB}]-I[\mathrm{~dB}] .
$$

The path loss will increase in (4), by decreasing the parameter $I$ while other parameters are remained fixed. By increasing the path loss while the frequency is fixed, the measure of $d$ or $R_{c}$ increases.

\subsection{Allocating the Best Channel}

Our objective is to maximize the number of simultaneous CR links and consequently maximizing the throughput of the whole network. At first, no specific frequency is allocated for CR transmission and it has to wait until one or more channels randomly become released, and it is absolutely against primary networks which have access to all channels without any limitation. At any time, one of the PUs may use the unoccupied channel and make it busy again for CRN. Therefore, applying the total capacity of the sub-channels and increasing the number of simultaneous links in CR network is an efficient way to increase the throughput of the network. This will increase the total throughput of the whole network [22], [24]. Reference [25] indicates that selecting the best sub-channel is an integer linear programming (ILP), which belongs to the class of NP-hard problem. This problem does not have a closed solution. In this paper, we propose a near-optimum and intelligent method to solve this problem, (NFRMAC) method. 


\section{Proposed Algorithm NFRMAC}

By assuming equal parameters (interference, SINR and $P_{t}$ ) for each channel, in the proposed method, larger frequencies are allocated to shorter distances and conversely, smaller frequencies are allocated to longer distances. Allocated frequency to each distance is called preferable channel list for a specific distance. In order to allocate channels by applying the mentioned method, each CR user divides its transmission zone to $h$ non-overlapping zones (concentric rings) while each zone has a preferable channel list $\varphi_{i}(\mathrm{~A}), i=1, \ldots, h$ so that the radius of the largest ring (zone) to the center of the node $\mathrm{A}$, is equal to $R_{c}$. Giving initial measures to $R_{c}$ is conducted by considering a fixed interference in (4), while $I=$ const. and consequently the path loss and $R_{c}$ are gained. The value of $R_{c}$ depends on the transmit power of node $P_{t}$, channel frequency, environmental noise and the measure of interference in each channel. By increasing the measure of $R_{c}$, the possibility of having more links with the condition of $d \leq R_{c}$ increases.

As a first innovation in the proposed method, when transmitting the packets, the transmitting node $\mathrm{A}$ sends an RTS message to the receiving node B to which it also transmits the channel list available to itself.

In addition to estimating the distance $d_{A B}$ and interference, the receiver node creates its preferable channel list $\varphi_{i}(\mathrm{~B})$ based on the distance between $\mathrm{A}$ and $\mathrm{B}$. If this list would be empty, it sends a message to the transmitter stating that it is not ready to receive data. If $\varphi_{i}(\mathrm{~B})$ consists of free frequencies, it sends this list through a CTS message to the transmitting node with an estimated distance and interference level. Also, it announces that it is ready to receive data. The transmitter compares the estimated distance with the channels' list and their maximum range. If the requested link distance would be less than $R_{c}$, data transmitting operation will be conducted, but in case it would be more than $R_{c}$, one more time, the transmitting node will calculate a new measure for $R_{C}\left(R_{C N E W}\right)$ using (4) and the estimated interference level. In fact, $R_{c}$ is updated for current conditions. If $R_{c N E W}>R_{c}$, and the requested link distance is less than $R_{C N E W}\left(d_{A B}>R_{C N E W}\right)$, then the link (connection) is established.

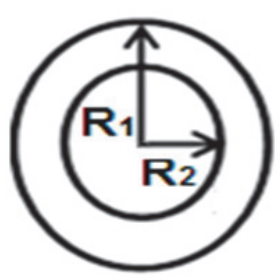

(a)

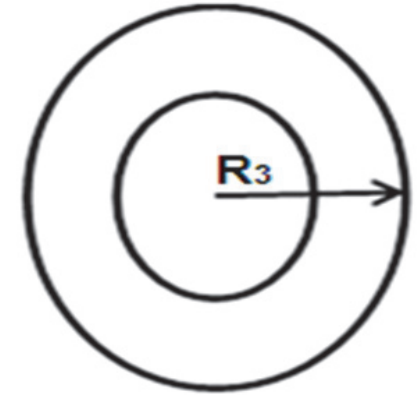

(b)
Fig. 2. DDMAC method and the proposed method are shown for two cases (a) and (b) respectively. The proposed method can cover a longer distance up to $R_{3}$ when decreasing the interference and noise.
Figure 2 shows the principle of the proposed method. In Fig. 2a, we have $R_{c}=R_{2}$ and in Fig. 2b, a situation is indicated that since estimated instantaneous interference is less than the fixed interference, the actual SINR is more than the threshold $\mu^{*}$ in the receiver, assuming other parameters to be fixed and with $\operatorname{SINR}=\mu^{*}$ by decreasing interference $I$ based on (4), a larger measure is obtained for the maximum acceptable path loss. In this case, assuming a larger $R_{c}$ such as $R_{3}>R_{2}$ is obtained. The increase of the distance is remarkable in this case.

\subsection{Pseudo Code of the Proposed Algorithm}

The proposed algorithm is described as below based on the step of correcting $R_{c}$ and also adding stop time to the random walk model. When a CR user (node A) seeks to transmit data to another CR user (node B) using our proposed algorithm, the following steps must be followed:

Step 1 - When node A has data packet, it transmits an RTS (Request to Send) message to node B. Also it sends its available channel list to node B.

Step 2 - The interference power level and the distance between the two nodes are estimated by the receiving node $\mathrm{B}$ and are reported to the transmitting node $\mathrm{A}$ through a control channel with common channels list.

Step 3 - If the common channel list is empty, go to step 7.

Step 4 - If the estimated distance is less than $R_{c}$, then transmit data. Go to step 8 .

Step 5 - Applying the estimated interference level, $\mu^{*}$ and (4), the transmitting node A calculates a new measure for $R_{c}$, called $R_{c N E W}$.

Step 6 - If the estimated distance is less than $R_{c N E W}$, then transmit data. Go to step 8 .

Step 7 - Establishing the requested link is impossible. Calculate blocking rate of transmission is not possible.

Step 8 - End.

By performing the aforementioned steps in order, packet transmission from node A to node B is performed. When data receiving is completed, an ACK message is transmitted from node B to node A. Flowchart of the proposed NFRMAC algorithm is presented in Appendix A, in Fig. A-1.

\section{Simulation Model}

In order to perform simulations, we have considered two primary networks and a $\mathrm{CR}$ network that all are located in the same geographic area. We consider a CRN that has 40 mobile nodes. There are 12 rings $(m=12)$ around a secondary node.

The node velocity in $\mathrm{CR}$ network varies between 0 and 6 meters per second. In this method, a node moves in a direction random with uniform distribution and then it 
stops for a fixed time ( $\left.t_{\text {pause }}\right)$, and then it moves again with different random direction and velocity in another direction similarly. Secondary users (nodes) produce packets for transmission based on a Poisson process with rate $\lambda$ (number of packets per time slot). Parameter $\lambda$ (means rate of packet transmission) is equal for all users. Sub-channels are considered to be active assuming a two-state Markovmodel for the active time duration and denoted by $\alpha_{i}$. Parameter $\alpha_{i}$ is defined as an activity factor of primary network. Three-stage link protocols of RTS, CTS and ACK are used for all links. A random walk model with stop time is used for moving CR nodes. Primary nodes are uniformly distributed in an area with size 100 by $100 \mathrm{~m}^{2}$. Out of 40 CR users, up to 20 can be uniformly selected as transmitters. Moreover, the number of packets produced by each $\mathrm{CR}$ user is randomly selected. We simulated the algorithm using Matlab on a core I7 CPU, 3.200 GHz, 4 GB RAM. Total required time for running was about 3 hours.

Using simulation results, the efficiency of the proposed NFRMAC method is compared with the reference methods: DDMAC and BMC. In our simulations, $\mu^{*}$ is considered to be equal to $5 \mathrm{~dB}$ and thermal noise density is $P_{t h}=10^{-21} \mathrm{Watt} / \mathrm{Hz}$, which is assumed to be equal for all channels. We compare throughput and packet blocking rate of the proposed protocol with those of the DDMAC and BMC. Communication blocking rate shows the packets not being delivered due to unavailability of idle channel in a special period of time. The two primary networks transmit in $900 \mathrm{MHz}$ and $2.4 \mathrm{GHz}$ band each having $1.5 \mathrm{MHz}$ bandwidth. We consider that primary network 1 has an activity factor $\alpha_{1}=0.5$ (meaning it is active $50 \%$ of time) and primary network 2 is active for $30 \%$ of time (i.e., $\alpha_{2}=0.3$ ). We also assume that the channel's status does not change when transmitting a data packet and three control packets. Moreover, the maximum transmit power is $P_{t}=0.5 \mathrm{~mW}$ in the proposed NFRMAC protocol and the DDMAC and BMC protocols. We considered 100 topologies for our simulation that each of them was conducted for 1000 seconds and finally the results indicated a superiority of proposed methods over previous methods. Simulation results are presented in Sec. 4.

\subsection{Simulation Results}

Figure 3 shows the normalized throughput versus the packet generation ratio for the proposed NFRMAC and DDMAC and BMC methods. We observe that the throughput increases by increasing packet generation ratio, and then it saturates so that the maximum normalized throughput of 1 is achieved. As can be seen in this figure, the proposed NFRMAC has about $6 \%$ more throughput compared with that of DDMAC.

Now, the blocking rate (in percentage) versus the packet generation ratio is shown in Fig. 4. We observe that the blocking rate of the proposed NFRMAC is up to $10 \%$ smaller than of the DDMAC and BMC when $\lambda=4$.

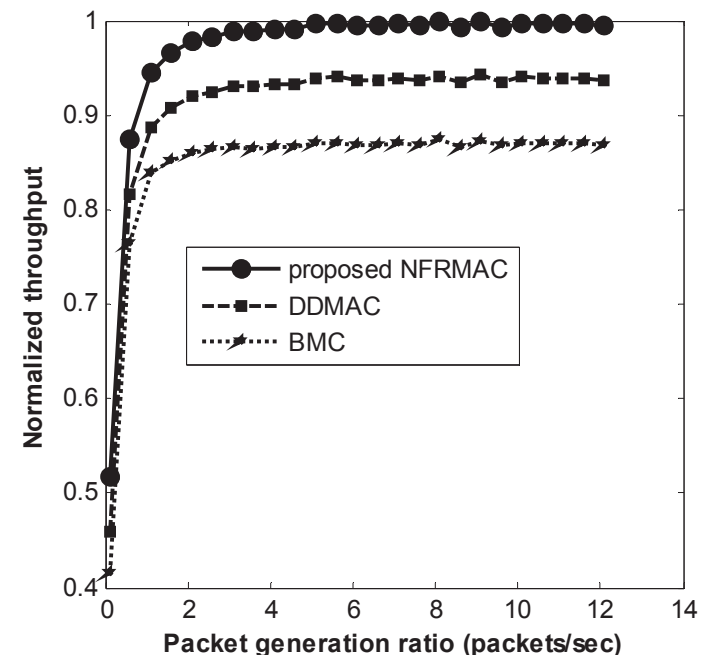

Fig. 3. Throughput versus packet generation ratio $(\lambda)$, for the proposed NFRMAC, and for the DDMAC and BMC methods.

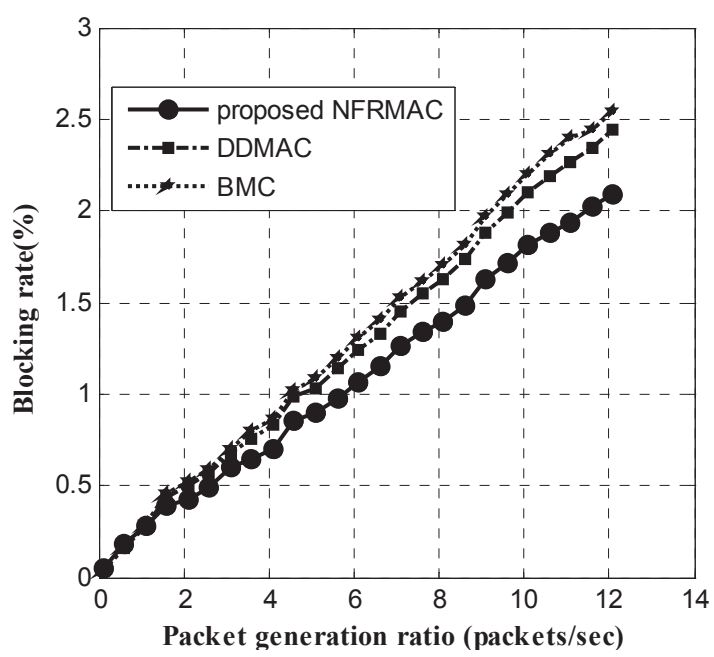

Fig. 4. Blocking rate versus packet generation ratio for the proposed NFRMAC and for the DDMAC and BMC methods.

\subsection{Effect of Node's Stop-time}

Figures 5 and 6 show the effect of node's stop-time. As mentioned before, this stop time is in fact a novelty in our model, which has not been considered so far by others. In Fig. 5, we analyze the effect of node stop time on the total throughput. We observe that on average, the proposed NFRMAC has a larger total throughput compared to those of the DDMAC and BMC.

Figure 6 shows the blocking rate versus the stop-time. We can observe that the proposed NFRMAC has a smaller blocking rate for different stop times.

It is worth mentioning that the oscillatory behavior of the throughput and blocking rate is due to the mobile nature of CR users, the probability of access to unoccupied channels, and the number of channels in a period of time. 


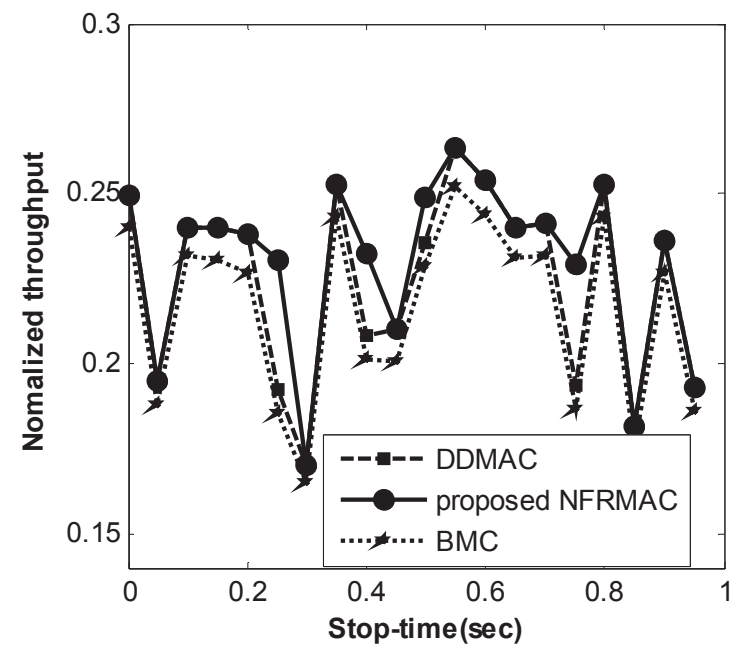

Fig. 5. Total throughput versus stop time of the nodes.

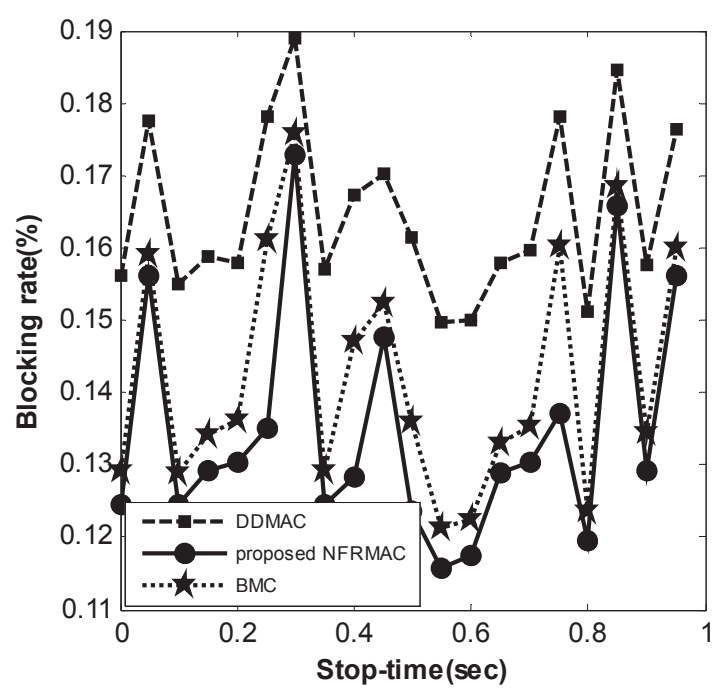

Fig. 6. Blocking rate of the packets versus stop time of nodes.

\subsection{Effects of the Number of CR Users}

Now, the effect of the number of CR users is analyzed on the network's throughput for the proposed NFRMAC and for the DDMAC and BMC methods. Figure 7 shows the achievable normalized throughput versus the number of $\mathrm{CR}$ users for the aforementioned three methods. As observed from Fig. 7, the proposed method and the DDMAC method performs similarly for up to 4 secondary users. However, when the number of $\mathrm{CR}$ users increases, the proposed NFRMAC outperforms both the DDMAC and BMC.

The packet blocking rate versus the number of $\mathrm{CR}$ users is shown in Fig. 8. As observed from this figure, the proposed method leads to less blocking rate in the network. Moreover, when the number of nodes increases up to more than 14 CR nodes, the blocking rate decreases slightly, this is due to the increase of the achieved throughput. By increasing the number of nodes, more connections are possible, and the increase in the throughput provided by our proposed NFRMAC is more noticeable after about $15 \mathrm{CR}$ users.

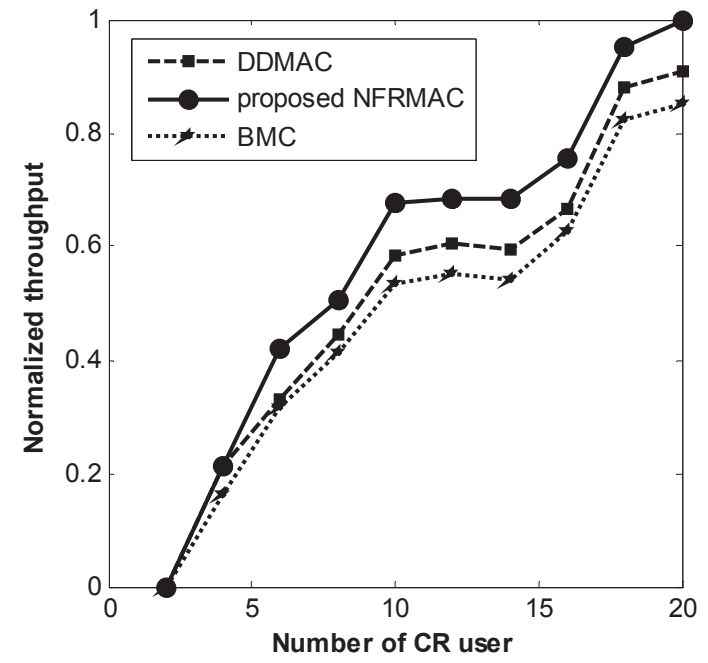

Fig. 7. Throughput versus the number of Cognitive Radios in the network.

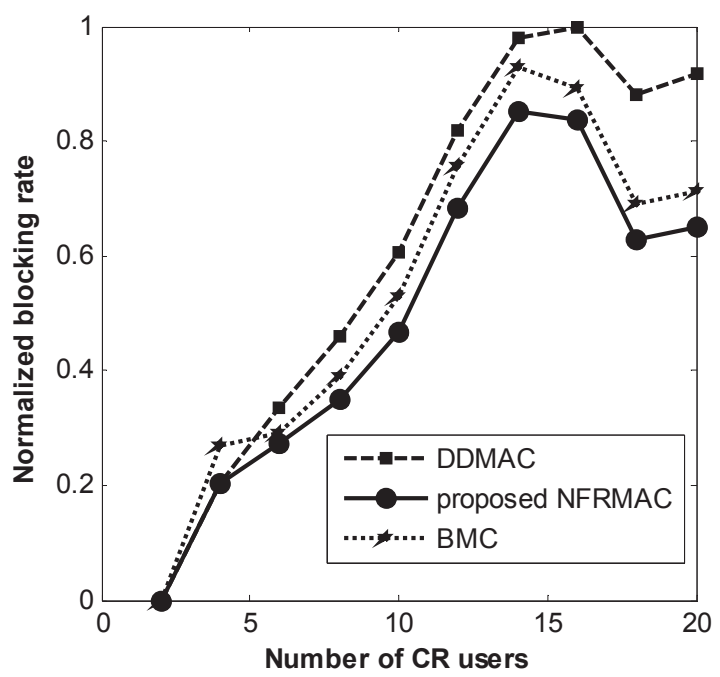

Fig. 8. Packet blocking rate versus the number of CR users.

\section{Conclusions}

In this paper, we proposed an opportunistic new frequency-range spectrum of MAC protocol for CRN in order to increase the achievable throughputs. The proposed NFRMAC method is in fact a new process for channel allocation, which creates a relation between attenuation model, signal frequency, transmission range, and interference level to increase the throughput of the CRN. In comparison with conventional methods, the NFRMAC adaptively maximizes the transmission range. We also presented a more realistic model for moving pattern of $\mathrm{CR}$ nodes in the NFRMAC by considering stop time instances for CR nodes. We showed that the proposed realistic model leads to an increase in the CRN throughput. More precisely, simulation results showed that the proposed method resulted in $6 \%$ throughput increase in comparison with that of the DDMAC method. Moreover, numerical results indicated that the NFRMAC exploits the spectrum more efficiently and decreases the communication blocking rate up to $10 \%$ compared to conventional methods. 


\section{Appendix}

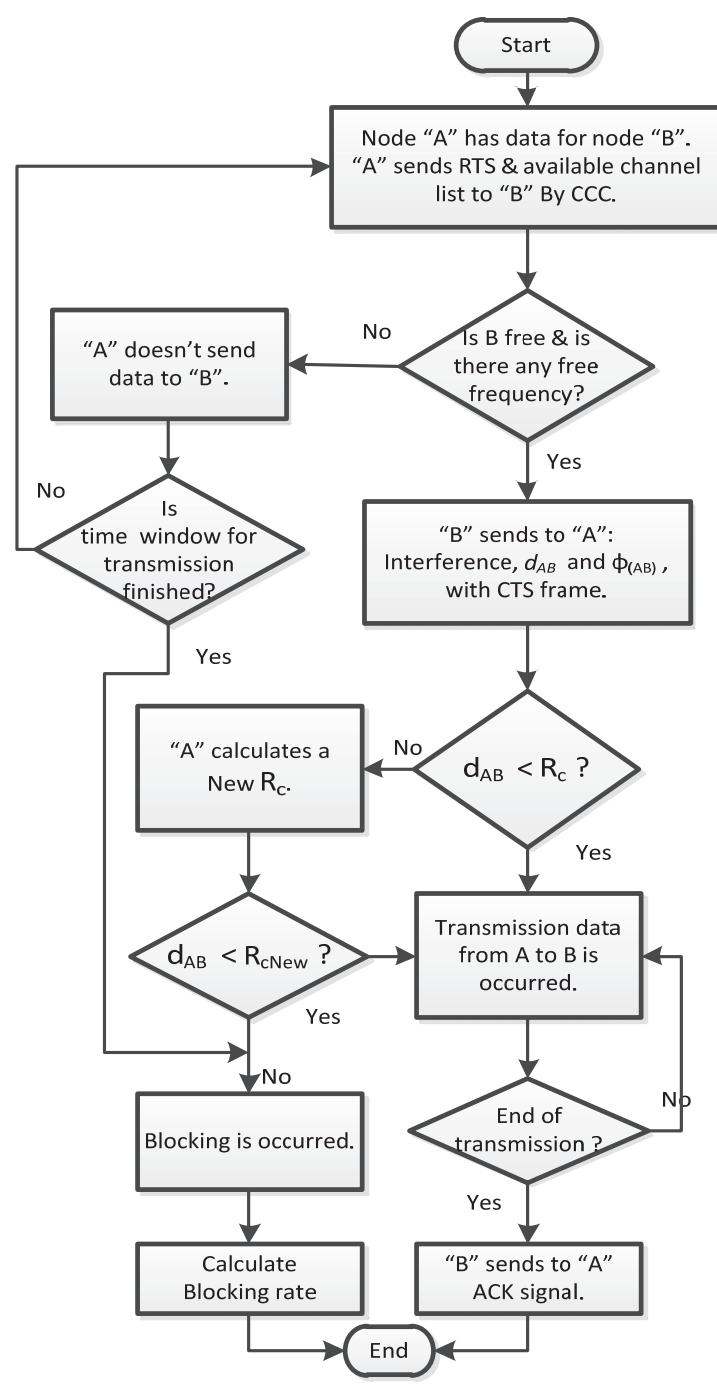

Fig. A-1. Flowchart of proposed NFRMAC algorithm.

\section{References}

[1] FCC Report of the Spectrum Efficiency Working Group. Federal Communications Commission Spectrum Policy Task Force, Nov. 2002.

[2] ZHANG, Z., WU, Q., Wang, J. Optimal energy-efficient cooperative spectrum sensing in cognitive radio networks. Radioengineering, 2013, vol. 22, no. 4, p. 1150-1155.

[3] AKYILDIZ, I. F., LEE, W. Y., VURAN, M. C., MOHANTY, S. A survey on spectrum management in cognitive radio networks. IEEE Communications Magazine, 2008, vol. 46, no. 4, p. 40-48. DOI: 10.1109/MCOM.2008.4481339

[4] AKYILDIZ, I. F., LEE, W. Y., VURAN, M. C., MOHANTY, S. $\mathrm{NeXt}$ generation/dynamic spectrum access/cognitive radio wireless networks: a survey. Computer Networks, 2006, vol. 50, no. 13, p. 2127-2159. DOI: 10.1016/j.comnet.2006.05.001

[5] ZHAO, Q., SADLER, B. M. A survey of dynamic spectrum access. IEEE Signal Processing Magazine, 2007, vol. 24, no. 4, p. 79-89. DOI: 10.1109/MSP.2007.361604
[6] CHEN, Y.-S., CHO, C.-H. YOU, I., CHAO, H.-C. A cross-layer protocol of spectrum mobility and handover in cognitive LTE networks. Simulation Modelling Practice and Theory, 2011, vol. 19, no.8, p. 1723-1744. DOI:10.1016/j.simpat.2010.09.007

[7] KASH, I., MURTY, R., PARKES, D. Enabling spectrum sharing in secondary market auctions. IEEE Transactions on Mobile Computing, 2014, vol. 13 , no. 3 , p. 556-568. DOI: 10.1109/TMC.2013.17

[8] LUO, T., LIN, F. JIANG, T., CHEN W. Multicarrier modulation And cooperative communication in multihop cognitive radio network. IEEE Wireless Communications, 2011, vol. 18, no. 1, p. 38-45. DOI: 10.1109/MWC.2011.5714024

[9] HAYKIN, S. Cognitive radio: brain-empowered wireless communications. IEEE Journal on Selected Areas in Communications, 2005, vol. 23, no. 2, p. 201-220. DOI: 10.1109/JSAC.2004.839380

[10] LIAO, S. H., CHIU, C. C., HO, M. H., LIU, C. L. Channel capacity of multiple-input multiple-output ultra-wideband systems with single co-channel interference. International Journal of Communication Systems, 2010, vol. 23, no. 12, p. 1600-1612. DOI: $10.1002 /$ dac. 1131

[11] KLOZAR, L., POLAK, L., KALlER, O., PROKOPEC. J. Effect of co-existence interferences on QoS of HSPA/ WCDMA mobile networks. In 23rd International Conference on Radioelektronika 2013. Pardubice (Czech Republic), 2013, p. 312-315. DOI: 10.1109/RadioElek.2013.6530937.

[12] NASIPURI, A., DAS, S. R. Performance of multichannel wireless ad hoc networks. International Journal of Wireless and Mobile Computing, 2006, vol. 1, no. 3, p. 191-203.

[13] SHU, T., CUI, S., KRUNZ, M. WLC05-3: medium access control for multi-channel parallel transmission in cognitive radio networks. In IEEE Global Telecommunications Conference GLOBECOM'06. San Francisco (CA, USA), 2006, p. 1-5. DOI: 10.1109/GLOCOM.2006.641

[14] ZHAO, Q., TONG, L., SWAMI, A. Decentralized cognitive mac for dynamic spectrum access. In 1st IEEE International Symposium on New Frontiers in Dynamic Spectrum Access Networks (DySPAN 2005). Baltimore (MD, USA), 2005, p. 224-232. DOI:10.1109/DYSPAN.2005.1542638

[15] NGUYEN-THANH, N., PHAM, A. T., NGUYEN, V.-T. Medium access control design for cognitive radio networks: A survey. IEICE Transactions on Communications, 2014, vol. E97-B, no. 2, p. 359-374. DOI: 10.1587/transcom.E97.B.359

[16] CLANCY, T. C. Achievable capacity under the interference temperature model. In 26th IEEE International Conference on Computer Communications (INFOCOM 2007). Anchorage (AK, USA), 2007, p. 794-802. DOI: 10.1109/INFCOM.2007.98

[17] BANY SALAMEH, H. A., KRUNZ, M., YOUNIS, O. Cooperative adaptive spectrum sharing in cognitive radio networks. IEEE/ACM Transactions on Networking, 2010, vol. 18, no. 4, p. 1181-1194. DOI:10.1109/TNET.2009.2039490

[18] JAIN, N., DAS, S. R., NASIPURI, A. A multichannel CSMA MAC protocol with receiver-based channel selection for multi-hop wireless networks. In Proceedings of the Tenth International Conference on Computer Communications and Networks. Scottsdale (AZ, USA), 2001, p. 432-439. DOI: 10.1109/ICCCN.2001.956301

[19] BetTSTETTER, H., HARENSTEIN, H., PEREZ-COSTA, X. Stochastic properties of the random waypoint mobility model. Wireless Networks, 2004, vol. 10, no. 5, p. 555-567. DOI: 10.1023/B:WINE.0000036458.88990.e5.

[20] BetTStetTer, C., REStA, G., SANTI, P. The node distribution of the random waypoint mobility model for wireless ad hoc networks. IEEE Transactions on Mobile Computing, 2003, vol. 2, no. 3, p. 257-269. DOI: 10.1109/TMC.2003.1233531 
[21] DANG, D. N. M., DANG, H. N., DO, C. T., HONG C. S. An enhanced multi-channel mac for vehicular ad hoc networks. In IEEE Wireless Communications and Networking Conference $(W C N C)$. Shanghai, 2013, 2013, p. 351-355. DOI: 10.1109/WCNC.2013.6554589.

[22] XU, K., GERLA, M., BAE, S. Effectiveness of RTS/CTS handshake in IEEE 802.11 based ad hoc networks. Ad Hoc Networks, 2003, vol. 1, no. 1, p. 107-123. DOI: 10.1016/S15708705(03)00015-5

[23] RAPPAPORT, T. S. Wireless Communications: Principles and Practice. Vol. 2. Prentice Hall PTR New Jersey, 2001.

[24] SIZUN, H. Radio Wave Propagation for Telecommunication Applications. Springer, 2005.

[25] ZHAO, H.S. ZHANG, J.Z., ZHUANG, W.H. A joint link and channel assignment routing scheme for cognitive radio networks. Radioengineering, 2013, vol. 22, no. 4, p. 1128-1137.

[26] ARIKAN, E. Some complexity results about packet radio networks (Corresp.). IEEE Transactions on Information Theory, 1984, vol. 30, p. 681-685. DOI: 10.1109/TIT.1984.1056928

\section{About the Authors...}

Seyed Ali Reza HOSSEINI KARRABI received his M.Sc. and B.Sc. degrees in Electrical Engineering (Telecommunication) from Tabriz and Shiraz University, Iran, in 1991 and 1997, respectively. He is currently a Ph.D. candidate in the Electrical Engineering School of Iran University of Science and Technology, Tehran, Iran. His research interests include mobile cognitive radio systems, wireless communications and signal processing.

Bahman ABOLHASSANI received the B.Sc. degree from Iran University of Science and Technology, Tehran, Iran in
1980, the M.S. and Ph.D. degrees from University of Saskatchewan, Canada in 1995 and 2001, respectively, all in Electrical Engineering. In 2002, he joined as an assistant professor to the Dept. of Electrical Engineering, IUST, Tehran, Iran. He served as the Head of Electrical Engineering School for two years. He is an associate professor and his research interests are in the areas of wireless communications, interference cancellation for CDMA systems, spread spectrum, wireless sensor networks, cognitive radio networks and resource allocation.

Seyed Mohammad Sajad SADOUGH received his B.Sc. degree in Electrical Engineering (Electronics) from Shahid Beheshti University, Tehran, I.R. Iran in 2002 and the M.Sc. and Ph.D. degrees in Electrical Engineering (Telecommunication) from Paris-Sud 11 University, Orsay, France, in 2004 and 2008, respectively. From 2004 to 2007, he held a joint appointment with the National Engineering School in Advanced Techniques (ENSTA), Paris, France, and the Laboratory of Signals and Systems (LSS), at Supélec, Gif-sur-Yvette, France. He was a lecturer in the Dept. of Electronics and Computer Engineering (UEI), ENSTA, where his research activities were focused on improved reception schemes for ultra-wideband communication systems. From December 2007 to September 2008, he was a postdoctoral researcher with the LSS, SupelecCNRS, where he was involved in the European research project TVMSL with Alcatel-Lucent France. Since October 2008, he has been a member of the Faculty of Electrical Engineering, Shahid Beheshti University, where he is currently an Assistant Professor in the Dept. of Telecommunication. Dr. Sadough's areas of research include signal processing, communication theory, and digital communication. 